\title{
CAVITATION IN SHOCK WAVE LITHOTRIPSY: THE CRITICAL ROLE OF BubBle ACTIVITY IN StONE BREAKAGE AND KidNEY TRAUMA
}

\author{
Michael R. Bailey ${ }^{1}$, Robin O. Cleveland ${ }^{2}$, Tim Colonius ${ }^{3}$, Lawrence A. Crum ${ }^{1}$, Andrew P. Evan ${ }^{4}$, James. E. \\ Lingeman $^{5}$, James A. McAteer ${ }^{4}$, Oleg A. Sapozhnikov ${ }^{6}$, and James C. Williams, Jr. ${ }^{4}$ \\ ${ }^{1}$ Center for Industrial and Medical Ultrasound, Applied Physics Laboratory, University of Washington, Seattle, \\ Washington, USA \\ ${ }^{2}$ Department of Aerospace and Mechanical Engineering, Boston University, Boston, Massachusetts, USA \\ ${ }^{3}$ Department of Mechanical Engineering, California Institute of Technology, Pasadena, California, USA \\ ${ }^{4}$ Department of Anatomy and Cell Biology, Indiana University School of Anatomy, Indianapolis, Indiana, USA \\ ${ }^{5}$ Methodist Hospital Institute for Kidney Stone Disease, Indianapolis, Indiana, USA \\ ${ }^{6}$ Department of Acoustics, Faculty of Physics, Moscow State University, Moscow, Russia
}

Abstract - OBJECTIVE: Shock Wave Lithotripsy (SWL) is the use of shock waves to fragment kidney stones. We have undertaken a study of the physical mechanisms responsible for stone comminution and tissue injury in SWL. SWL was originally developed on the premise that stone fragmentation could be induced by a short duration, high amplitude positive pressure pulse. Even though the SWL waveform carries a prominent tensile component, it has long been thought that SW damage to stones could be explained entirely on the basis of mechanisms such as spallation, pressure gradients, and compressive fracture. We contend that not only is cavitation also involved in SWL, bubble activity plays a critical role in stone breakage and is a key mechanism in tissue damage. METHODS: Our evidence is based upon a series of experiments in which we have suppressed or minimized cavitation, and discovered that both stone comminution and tissue injury is similarly suppressed or minimized. Some examples of these experiments are (1) application of overpressure, (2) time reversal of acoustic waveform, (3) acoustically-transparent, cavitation-absorbing films, and (4) dual pulses. In addition, using passive and active ultrasound, we have observed the existence of cavitation, in vivo, and at the site of tissue injury. RESULTS: Numerical and experimental results showed mitigation of bubble collapse intensity by time-reversing the lithotripsy pulse and in vivo treatment showed a corresponding drop from $6.1 \% \pm 1.7 \%$ to $0.0 \%$ in the hemorrhagic lesion. The time-reversed wave did not break stones. Stone comminution and hemolysis were reduced to levels very near sham levels with the application of hydrostatic pressure greater than the near $10-\mathrm{MPa}$ amplitude of the negative pressure of the lithotripter shock wave. A Mylar sheet 3-mm from the stone surface did not inhibit erosion and internal cracking, but a sheet in contact with the stone did. In water, mass lost from stones in a dual pulse lithotripter is 8 times greater than with a single lithotripter, but in glycerol, which reduces the pressures generated in bubble implosion, the enhancement is lost. CONCLUSION: This cavitation-inclusive mechanistic understanding of SWL is gaining acceptance and has had clinical impact. Treatment at slower SW rate gives cavitation bubble clusters time to dissolve between pulses and increases comminution. Some SWL centers now treat patients at slower SW rate to take advantage of this effect. An elegant cavitation-aware strategy to reduce renal trauma in SWL is being tested in experimental animals. Starting treatment at low amplitude causes vessels to constrict and this interferes with cavitationmediated vascular injury. Acceptance of the role of cavitation in SWL is beginning to be embraced by the lithotripter industry, as new dual-pulse lithotriptersbased on the concept of cavitation control- have now been introduced.

\section{INTRODUCTION}

Shock wave lithotripsy (SWL) is a common and effective clinical method to comminute kidney stones. However, the evidence is increasingly strong that SWL treatment also causes tissue injury, and that by many clinical measures, lithotripsy is becoming neither more efficacious nor safer. Comminution studies fail to show that newer tight-focal machines break stones more efficiently than the first lithotripter design to gain widespread clinical use in the 1980 s (Dornier HM-3) [1]. Retreatment rates are higher with new machines [2]. Incidence of adverse effects 
is increasing [2]. Comminuting ureteral stones remains difficult. We conclude from this current state of lithotripsy that there is a desire and a need for a fuller understanding of the mechanisms of tissue damage and stone comminution and for direction on using this understanding to improve treatment.

\section{RESULTS}

\section{Overpressure}

Elevated hydrostatic pressure (overpressure) suppresses stone comminution, reduces cavitationpitting on aluminum foil, and dramatically reduces cell lysis in vitro [3]. Figure 1 shows some results. The inset shows the chamber which has an acoustically matched window for efficient transmission of the shock wave. Area increase is the

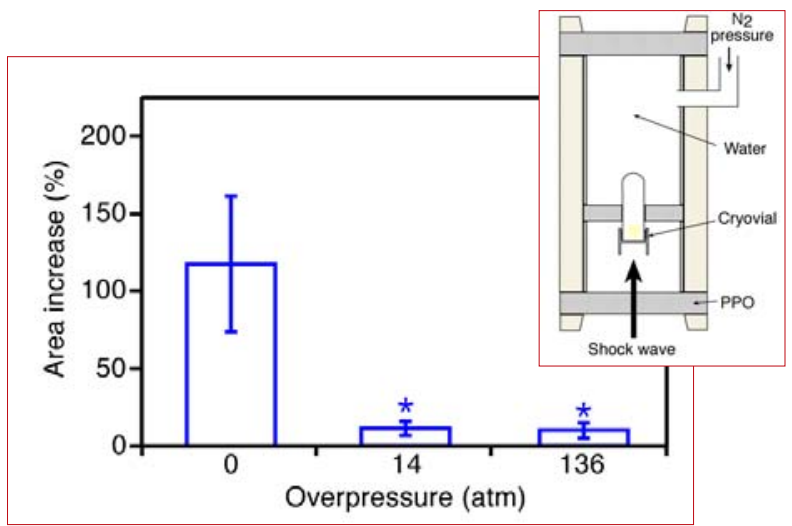

Figure 1. Stone comminution is suppressed by elevating the static pressure. Overpressure's effect is to dissolve cavitation nuclei.

projected area of the stone fragments on a flat digital scanner relative to the projection of the intact stone. This method allows for rapid quantification of comminution without drying (i.e., introducing gas into) the stone. Comminution effectiveness is dramatically reduced at both 14 and 136 atm of static pressure although the negative pressure of the lithotripter shock wave is 100-120 atm. Lysis of suspended cells drops from $10 \%$ to near control levels $(<1 \%)$ at only $1-2 \mathrm{~atm}$ of overpressure.

\section{Cavitation Interference}

Stones did not break when placed in a viscous liquid [4] and even a small membrane placed against the surface of the stone prevented cavitation collapse on the surface and reduced comminution [5]. Figure 2 shows the results of surrounding the stone in watersoaked gauze as a tissue phantom and placing a thin
Mylar disk either against or a few $\mathrm{mm}$ from the proximal surface of the stone [6]. The stones are cylindrical cement stones. The top are $\mu \mathrm{CT}$ images through the stones along the SW axis. The bottom images show damage to the stone's proximal face. This model cement stone implanted into a pig ureter also fails to fragment as well as stones implanted into the pig collecting system. Clinically, ureteral stones are very difficult to break. Our hypothesis is that the close contact with the ureter inhibits the formation of a cavitation cluster and violent collapse of the cluster on the stone surface. In contrast in a cavitation promoting medium, tissue injury is increased. Injection of microbubbles into the blood stream before lithotripsy significantly increased renal tissue injury [7].

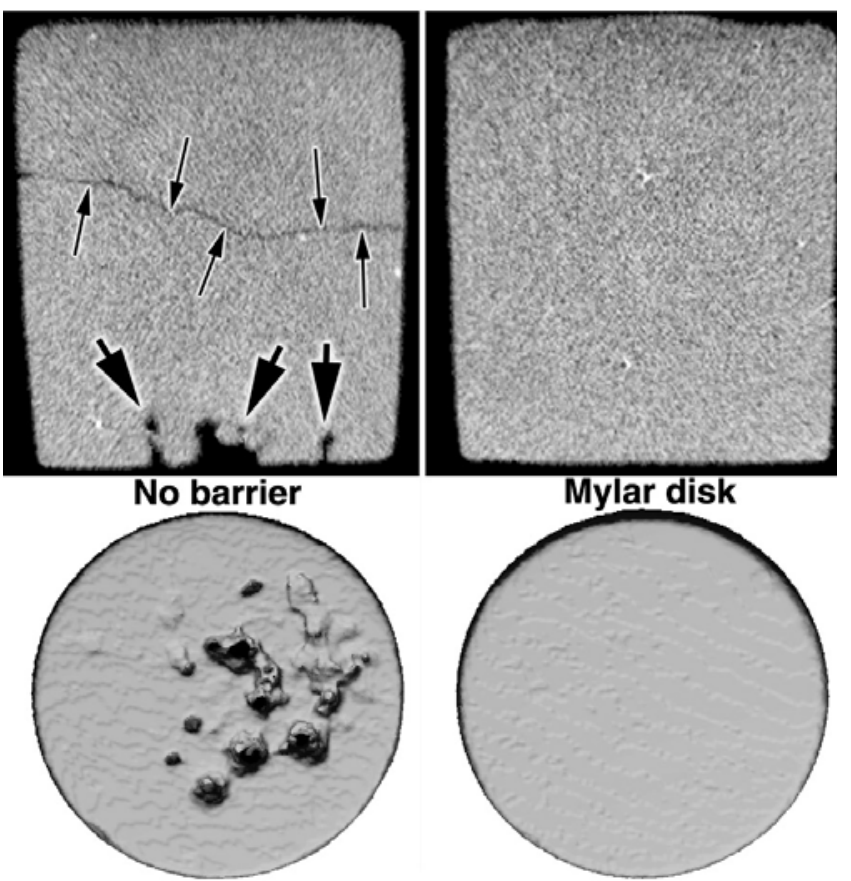

Figure 2. Damage to stones without (left and with (right) a Mylar film in contact with the proximal surface. Erosion and a fracture line (arrows) are apparent on the left but not right.

\section{Stone Location}

The peak negative pressure in the Dornier HM3 lithotripter occurs $2 \mathrm{~cm}$ proximal to the geometric focus of the ellipsoid where the stone is placed for treatment. The peak positive is $\sim 1 \mathrm{~cm}$ distal. The difference in focusing is attributed to self-refraction and has been calculated and measured. Suitably the maximum cavitation activity (measured by a freeradical chemical reaction) in vitro is greatest at the -2 
$\mathrm{cm}$ position [8]. Stone comminution and hemolysis in vitro are also greatest at the $-2 \mathrm{~cm}$ position [8]. Figure 3 shows the stone results.

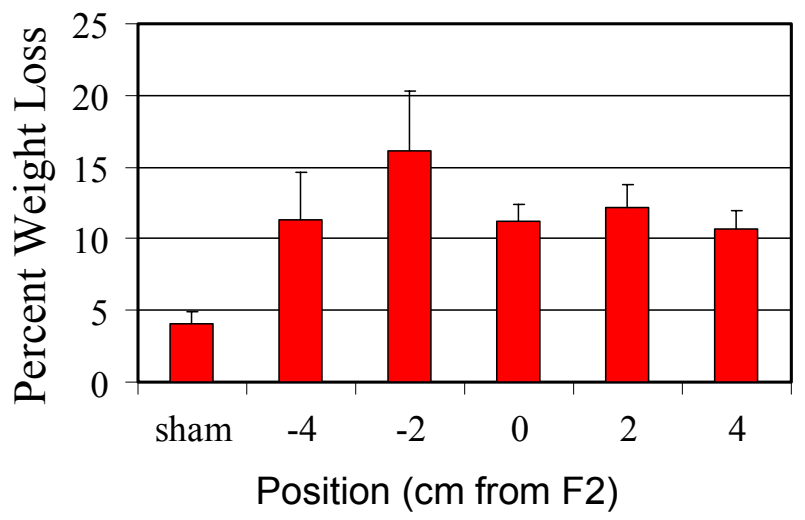

Figure 3. Stone comminution in vitro is significantly better at $-2 \mathrm{~cm}$ where cavitation activity and peak negative pressure are greatest.

\section{Waveform Manipulation}

Dramatically different cavitation was produced by two separate acoustic pulses that had different shapes but similar duration, frequency content, and peak positive and negative pressure [9]. Both pulses were produced by a Dornier HM-3 style lithotripter: one pulse when the ellipsoidal reflector was rigid, the

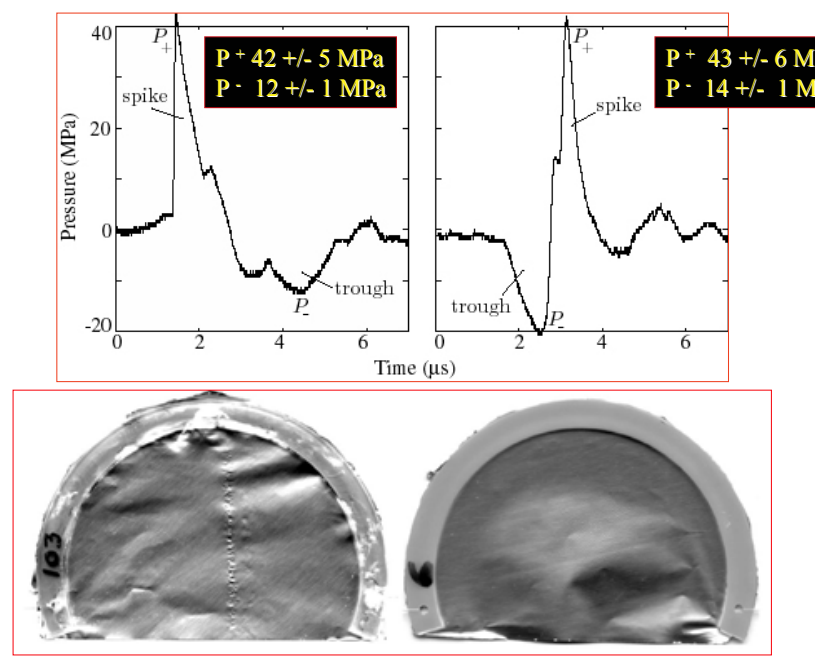

Figure 4 . The conventional lithotripter produced by the rigid reflector (left) and the essentially timeinverted waveform produced by the pressurerelease reflector (right). The inset shows pitting at the focus and along the axis of the rigid reflector (left) and negligible pitting at the focus and along the axis of the pressure-release reflector (right). other when the reflector was pressure release. Figure 4 shows sample waveforms and pits in foil from one pulse with each reflector. The cavitation, or bubble action, generated by the conventional rigid-reflector pulse was nearly 50 times longer lived and 3-13 times stronger than that produced by the pressure-releasereflector pulse. Cavitation durations measured by passive acoustic detection and high speed video agreed with calculations based on the Gilmore equation. Cavitation intensity, or destructive potential, was judged (1) experimentally by the size of pits in aluminum foil detectors and (2) numerically by the calculated amplitude of the shock wave emitted by a collapsing bubble. A line of pits is clear when the rigid reflector is used but cannot be seen when the pressure-release reflector is used. Pit depth is decreased by a factor of 3 and pit width decreased by 7 . The pressure generated in the bubbles as calculated using the Gilmore code is decreased 13fold with the pressure-release reflector waveform. Our results indicate that the trailing positive spike in the pressure-release-reflector waveform stifles bubble growth and mitigates the collapse, whereas, the trough after the positive spike in the rigid-reflector waveform triggers inertially driven growth and collapse. The two reflectors therefore provide a tool to compare effects in weakly and strongly cavitating fields and thereby help assess the role of cavitation in lithotripsy.

The pressure-release reflector essentially eliminated tissue injury in pigs. Pig kidneys treated with shock waves from a standard rigid reflector showed damage that was evident even on gross inspection as a subcapsular bleed consistently at the treated pole (lower pole) of the kidney but frequently extended to portions of the upper pole as well. In comparison, very little damage occurred to kidneys treated with the PRel reflector [10]. These kidneys did not develop hematomas. On histological analysis the renal cortex was virtually undamaged and evidence of injury was limited to slight intraparenchymal bleeding in renal papillae located within the focal zone of the lithotripter. Such regions of bleeding were only visible in high-magnification histological sections and these were too slight to register by quantitative morphometric analysis. The PRel reflector also suppressed stone comminution (CaOx human stones, $18 \mathrm{kV}, 75 \mathrm{SWs}$ in vitro). No measurable comminution was recorded with the PRel reflector whereas stones were well fragmented with 
the rigid reflector. Thus cavitation appears to be very important in stone comminution and tissue injury and the lithotripter waveform can be used to control both effects.

\section{CONCLUSIONS}

In conclusion, there is a wealth of data showing that cavitation plays a significant role in shock wave lithotripsy. Cavitation is clearly involved in stone breakage and there is solid evidence linking bubble activity with SWL-induced tissue damage. Strategies that control cavitation - pulse repetition rate, elevated static pressure, alteration of the fluid medium around the stone, waveform manipulation, and the time delay between dual pulses -- all have a significant effect on stone comminution and tissue injury. Thus, the potential exists to use cavitation control techniques to engineer better lithotripters and to improve how lithotripsy is performed.

The wealth of data on the role of cavitation in SWL is having clinical impact as dual-pulse lithotripters are emerging and some clinicians are slowing treatment rate, but engineering solutions and treatment protocols to enhance efficacy and improve the safety of SWL would greatly benefit from further research. For example, questions remain such as how can "beneficial" cavitation that causes stone comminution be separated from "deleterious" cavitation that causes tissue injury? Or why does slower rate improve stone comminution [2]? Detailed models corroborated by in vitro and in vivo experiment and focusing on the collective effects of bubble clusters have the potential to answer these questions and to further improve clinicians' and manufacturers' abilities to control cavitation for safer, more efficacious lithotripsy.

\section{ACKNOWLEDGMENTS}

We gratefully acknowledge support from NIH grant \# DK43381, \#DK55674, and FIRCA and NSBRI SMS00203. We thank all the members of the Consortium for Shock Waves in Medicine for their contributions.

\section{REFERENCES}

1. Teichman, JMH, Portis, AJ, et al, "In vitro comparison of shock wave lithotripsy machines," J. Urol. 164, 1259-1264, 2000.
2. A. P. Evan, J. A. McAteer, J. C. Williams, Jr., L. R. Willis, M. R. Bailey, L. A. Crum, J. E. Lingeman, and R. O. Cleveland, "Shock wave physics of lithotripsy: Mechanisms of shock wave action and progress toward improved SWL," in Minimally Invasive Urology, (in press).

3. McAteer JA, M. A. Stonehill, K. Colmenares, J. C. Williams, Jr., A.P. Evan, R. O. Cleveland, M. R. Bailey, and L. A. Crum, "SWL cavitation damage in vitro: Pressurization unmasks a differential response of foil targets and isolated cells," J. Acoust. Soc. Am. 103, 3038 (A) and in Proc. of 16th International Congress on Acoustics (Seattle, Washington, USA, 1998), 2497-2498. (1998).

4. N. Vakil, S. M. Gracewski, and E. C. Everbach, "Relationship of model stone properties to fragmentation mechanisms during lithotripsy," J. Lithotripsy \& Stone Disease 3(4) 304-310 1991.

5. Holmer NG, Almquist LO, Hertz TG et al. On the mechanism of kidney stone disintegration by acoustic shock waves. Ultrasound Med Biol 17 479-489 1991.

6. McAteer JA, Cleveland RO, Rietjens, DL, et al. Cavitation promotes spall failure of model kidney stones treated by shock wave lithotripsy in vitro. Proc 17th Int Congr Acoust; Vol. 7, pp. 188-189, 2002.

7. Dalecki D, C. H. Raeman, S. Z. Child, D. P. Penney, R. Mayer, and E. L. Carstensen, "The influence of contrast agents on hemorrhage produced by lithotripter fields," Ultrasound Med. Biol. 23, 1435-1439 (1997).

8. Sokolov DL, M. R. Bailey, and L. A. Crum, "Stone Alignment in the Dornier HM3: The Role of Cavitation," J. Endourology 16 709-15 (2002).

9. Bailey MR, "Control of acoustic cavitation with application to lithotripsy," Technical Report ARL-TR-97-1, Applied Research Laboratories, The University of Texas at Austin, Austin, Texas and Defense Technical Information Center, Belvoir, Virginia (1997).

10. A. P. Evan, L. R. Willis, B. A. Connors, Y. Shao, J. E. Lingeman, J. C. Williams, Jr., J. A. McAteer, N. S. Fineberg, M. R. Bailey, and L. A. Crum, "Kidney damage and renal functional changes are minimized by waveform control that suppresses cavitation in SWL," J. Urol., 168(4 Pt. 1), 1556-62 (2002). 